\title{
Characterization of the Male-sterile Line Bcajh97-01 A/B and Identification of Candidate Genes for Genic Male Sterility in Chinese Cabbage-pak-choi
}

\author{
Li Huang, Wan-zhi Ye, Ting-ting Liu, and Jia-shu Cao ${ }^{1}$ \\ Laboratory of Cell and Molecular Biology, Institute of Vegetable Science, Zhejiang University/Key \\ Laboratory of Horticultural Plant Growth, Development and Quality Improvement, Ministry of \\ Agriculture, 268 Kaixuan Road, Hangzhou, 310029, China
}

\begin{abstract}
AdDitional INDEX wORDs. Brassica campestris, flower buds, GMS, transcriptional profiling, cDNA-AFLP
Abstract. Cytological features of 'Aijiaohuang' chinese cabbage-pak-choi (Brassica campestris ssp. chinensis) Bcajh97-01A/B genic male-sterile $A B$ line were examined to determine phenotypic reasons for male sterility. The sterile line Bcajh97-01A was found to undergo aberrant cytokinesis during male meiosis. Transcriptional profiling of the flower buds of both fertile and sterile plants was performed at the periods preceding meiosis, at the tetrad to uninucleate pollen period, and at the binucleate to mature pollen period. Transcript-derived fragments (TDFs) from corresponding genes that were expressed in flower buds at these three different stages could be divided into nine classes. We sequenced a total of 14 new TDFs that were differentially displayed at particular pollen developmental stages, including eight genes with unknown or hypothetical functions and six genes showing significant homology with known genes. This characterization of the Bcajh97-01A genic male-sterile line allowed the identification of candidate genes underlying genic male sterility.
\end{abstract}

The use of male-sterile plants has become an important technique in heterosis breeding to simplify and reduce the cost of seed production. Heterosis is used often in the breeding of cruciferous crops, with male-sterile lines providing a useful system for hybrid seed production. For example, various malesterile types isolated from Brassica napus, Brassica oleracea, and Brassica campestris (syn. Brassica rapa) are now widely applied in agricultural production.

The mechanism underlying male sterility is not yet clear, although considerable advances have recently been made in understanding male sterility at the molecular biology level. Many reviews of this research as well as various opinions and hypotheses on male sterility have been published (Araya et al., 1998; Hanson and Bentolila, 2004). However, current findings are still not sufficient to fully explain the male sterility phenomenon.

In the past, genic male-sterile cruciferous plants were considered worthless, because the $\mathrm{AB}$ lines developed from the genic male-sterile plants comprised only $50 \%$ sterile plants. The remaining $50 \%$ male-fertile plants had to be discarded during the production of $\mathrm{F}_{1}$ hybrids. However, chinese cabbage (B. campestris ssp. pekinensis) demonstrated interactive inheritance of genic male sterility, which allowed development of a male-sterile line with $100 \%$ sterile plants. This type of sterility was at first assumed to be controlled by the interaction of two pairs of genes (Wei et al., 1992; Zhang et al., 1990), but later was interpreted as inheritance-controlled by multiple alleles, and a corresponding "multiple allele model for genic male sterility" was proposed (Feng et al., 1996). Thus, male-

Received for publication 19 Aug. 2009. Accepted for publication 23 Oct. 2009. This work was supported by the Natural Science Foundation of China (Nos. 30871715 and 30800697).

${ }^{1}$ Corresponding author. E-mail: jshcao@zju.edu.cn. sterile plants produced by the interaction of multiple nuclear genes potentially can develop into stable genic male-sterile lines. For this reason, isolation of the candidate genes imparting genic male sterility and knowledge of the effects of these genes are important for the understanding of the mechanism of plant male sterility.

In a previous test-crossing study, we were able to produce the genic male-sterile AB line Bcajh97-01A/B from 'Aijiaohuang' chinese cabbage-pak-choi (B. campestris ssp. chinensis). The line showed stable genetic characteristics and was used in hybrid seed production during the 1980s to 1990s (Cao and $\mathrm{Li}$, 1980). The male-sterile line Bcajh97-01A lacks mature pollen grains in the anther but has otherwise normal flower development, resembling that of the fertile line Bcajh97-01B. Sterility is controlled by a recessive mutation at a single nuclear locus (Huang et al., 2008c), making this line excellent for investigating the mechanism of genic male sterility. Comparison of genomewide transcriptional profiles of the flower buds of fertile and sterile plants has revealed many genes associated with male sterility (Huang et al., 2008c; Wang et al., 2005; Ye et al., 2003). Some of these genes are clearly involved in pollen development (Cao et al., 2006; Huang et al., 2007, 2008a, 2008b, 2009a, 2009b; Zhang et al., 2008). However, the reason for failure of pollen formation is still unknown, and this has become a bottleneck to further study and subsequent use of this line.

Cytological observations on pollen development are useful for determining the characteristics of pollen abortion and the time at which abortion occurs (Laser and Lersten, 1972). These types of studies may complement findings at the molecular level, helping to explain the action of the expressed genes in relation to pollen abortion. Therefore, in the present study, an in-depth cytological study and comparison was carried out on the phenotypic features of male-sterile plants. To identify more 
candidate genes related to pollen abortion, we conducted parallel cDNA-amplified fragment length polymorphism (cDNA-AFLP) analysis to compare cytology and gene expression in fertile and sterile flower buds at the following three developmental stages: Stage 1, the period preceding meiosis; Stage 2, the tetrad to uninucleate pollen period; and Stage 3, the binucleate to mature pollen period.

\section{Materials and Methods}

Plant materials. During the 1980s, a male-sterile mutant lacking mature pollen, Brassica campestris male sterile (bcms), was identified in chinese cabbage-pak-choi. The female function of the mutant was unaffected and could be maintained by fertilization with normal pollen. When the mutant was crosstested with different male fertile plants, some $\mathrm{F}_{1}$ populations contained both male-sterile and male-fertile plants with segregation in a 1:1 ratio after $\chi^{2}$ analysis $(P>0.5)$. These progenies were developed into the genic male-sterile system named Bcajh97-01, which contains two sibling (sister) lines (the sterile Bcajh97-01A and the fertile Bcajh97-01B). The plants were cultivated in the experimental farm of Zhejiang University, Hangzhou, China.

Cytological examination. Buds of different sizes were fixed in FAA [38\% (v/v) formaldehyde:acetic acid:50\% (v/v) ethanol $=1: 1: 18]$, stored in $70 \%(\mathrm{v} / \mathrm{v})$ ethanol, and dehydrated through an ethanol series up to $70 \%(\mathrm{v} / \mathrm{v})$ ethanol and then embedded in paraffin. Five-micron-thin sections were stained with ferrovanadium and hematoxylin and examined with a microscope (DMLB; Leica, Wetzlar, Germany).

Transmission ELECTRON MiCROSCOPY. Flower buds or anthers were fixed overnight with $2.5 \%$ (v/v) glutaraldehyde, which contained $0.01 \%(\mathrm{w} / \mathrm{v})$ polyoxyethylene sorbitan monolaurate, rinsed in $0.1 \mathrm{M}$ phosphate buffer, and transferred to $1 \%$ (w/v) osmic acid for $1 \mathrm{~h}$. After an additional washing in phosphate buffer, specimens were dehydrated through an ethanol series up to $80 \%$ (v/v) ethanol and embedded in Spurr's resin. Ultrathin sections were stained with uranyl acetate and lead citrate and viewed with an electron microscope (JEM1230; JEOL, Tokyo, Japan) operated at $80 \mathrm{kV}$.

RNA EXTRACTION AND CDNA sYNTHESIS. Total RNA was extracted from different tissues of Bcajh97-01A and Bcajh9701B plants using Trizol reagent (Gibco, Berlin, Germany). Poly (A) ${ }^{+}$RNA was isolated from the total RNA with an Oligotex mRNA Mini Kit (Qiagen, Hilden, Germany). The first and double-stranded cDNA was then synthesized using a SMART ${ }^{\mathrm{TM}}$ cDNA Library Construction Kit (Clontech, Mountain View, $\mathrm{CA}$ ) according to the manufacturer's instructions. The longdistance polymerase chain reaction (PCR) products were purified using a QIAquick PCR Purification Kit (Qiagen).

CDNA-AMPLIFIED FRAGMENT LENGTH POLYMORPHISM. A cDNA-AFLP analysis was performed according to the procedures described by Bachem et al. (1996). A double-strand cDNA was digested with Taq I and Ase I as restriction enzymes. Selective amplification products were separated on a $6 \%$ polyacrylamide gel run at $1500 \mathrm{~V}$ until the xylene cyanole reached the bottom. DNA fragments were visualized by silver staining according to the Silver Sequencer DNA Sequencing System Technical Manual (Promega, Madison, WI). The differential expression patterns were determined using the Image Quant TL software V2003.02 (Pharmacia Biosciences, Piscataway, NJ), and for differential display analysis, two rounds of cDNA-
AFLP were conducted with two independently isolated total RNA samples.

ISOLATION AND SEQUENCING OF FRAGMENTS. The bands of interest were marked, cut out with a surgical blade, and incubated in $100 \mu \mathrm{L}$ of TE $(10 \mathrm{~mm}$ Tris, $\mathrm{pH} 7.5$, and $1 \mathrm{~mm}$ EDTA, $\mathrm{pH}$ 8.0) overnight at $37^{\circ} \mathrm{C}$. The AFLP fragments were recovered by PCR under the same conditions used for the preamplification. The reamplified cDNAs were subcloned using the pGEM-Teasy vector system (Promega) and sequenced using the ABI Prism 377 and 3730 sequencers from Invitrogen Biotech (Shanghai, China). Database searches were performed using the basic local alignment search tool (BLAST) network service. Each transcript-derived fragment sequence was compared against all sequences in the nonredundant database using the BLASTX and BLASTN program, and the sequences that returned with no significant homology were again compared against the expressed sequence tag databases using the BLASTN program. Program default parameters were used for all analyses.

REVERSE TRANSCRIPTASE-POLYMERASE CHAIN REACTION ANALYSIS. The total RNAs and first-stranded cDNA were prepared as described previously. Two rounds of reverse transcriptase (RT)-PCR were conducted with two independently isolated total RNA samples as templates. The RT-PCR was performed for $18,23,28,33$, and 38 cycles to determine the linearity of the PCR, and the thermal cycling parameters used for all genes were set as follows: $94^{\circ} \mathrm{C}$ for $30 \mathrm{~s}$, annealing for $30 \mathrm{~s}$ (the temperature varied with specific primers), and $72^{\circ} \mathrm{C}$ for $30 \mathrm{~s}$ for a total of 28 cycles. A 350-bp actin-1 fragment was amplified under the same RT-PCR conditions for a total of 28 cycles using the primer pair 5' - TCTCTATGCCAGTGGTCGTA-3' and 5'-CCTCAGGAC AACGGAATC-3' as a positive control. The gene specificity of the RT-PCR products was then confirmed by sequencing.

\section{Results}

The MALe-STerile line BCAJH97-01A has a DEFeCt IN MALE MEIOTIC CYTOKINESIS. Before the anaphase II stage, the cytology of pollen development in Bcajh97-01A (Fig. 1A) was not significantly different from that of the fertile Bcajh97-01B plant (Fig. 1B). However, at telophase II, the microsporocytes were loose and irregular, although they contained four nuclei (Fig. 1C). In Bcajh97-01B, the microsporocytes became enclosed in a thick wall of callose and the cytoplasm appeared concentrated (Fig. 1D). No tetraspores were formed in Bcajh97-01A as evidenced by the absence of intersporal walls in tetrads (Fig. 1E). Subsequently, an abundance of grain-like matter appeared, but in contrast with the smooth round uninucleate microspore of Bcajh97-01B (Fig. 1H), the grain-like matter in Bcajh97-01A did not enclose granular cytoplasm or a centrally placed nucleus (Fig. 1G). The pollen sacs began to fill with cytoplasm (Fig. 1I), but this gradually disintegrated, leaving the final pollen sacs empty (Fig. 1K). In contrast, the Bcajh97-01B pollen sacs at the same stage were full of mature pollen grains (Figs. 1J and 1L). Chromosome behavior during microsporegenesis in Bcajh9701A also revealed no tetraspore stage in Bcajh97-01 A, although the stages of diakinesis, metaphase I, anaphase I, telophase I, metaphase II, anaphase II, and telophase II were normal during the process of male meiosis (L. Huang, unpublished data). Meiosis appeared to be the critical period of pollen development that was perturbed in Bcajh97-01A, whereas karyokinesis appeared not to be affected. 


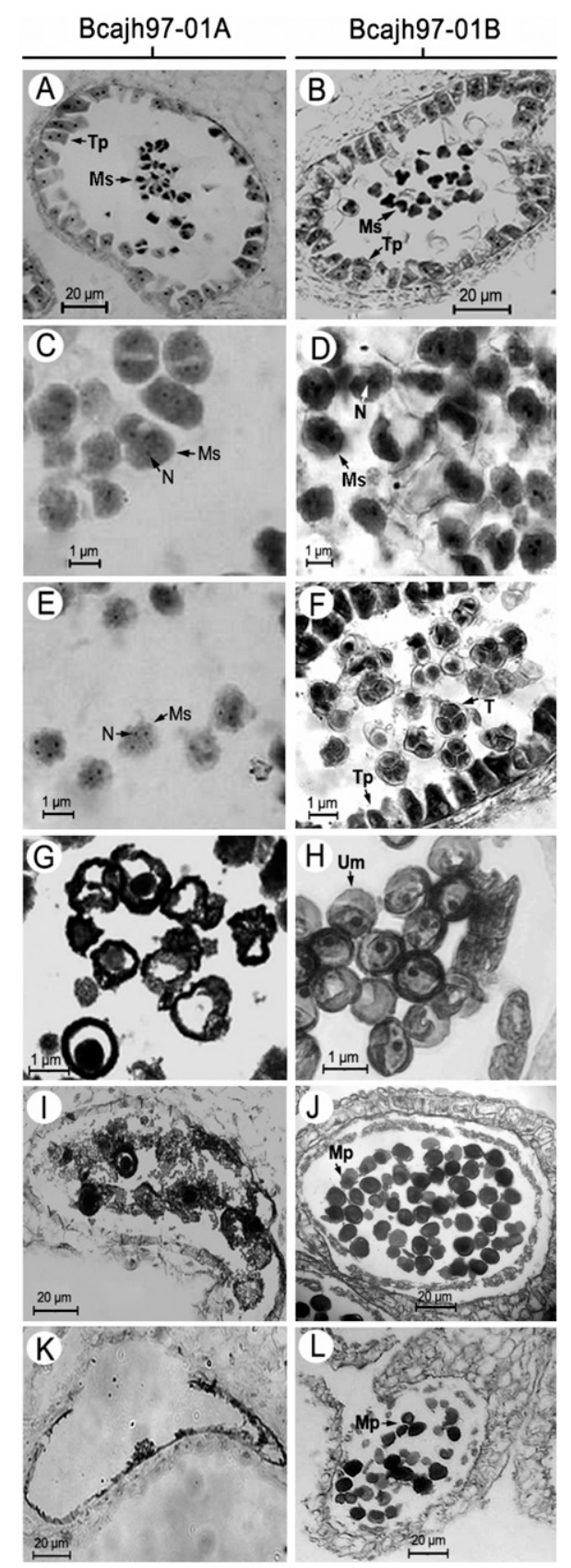

Fig. 1. Cytology of pollen and anther development of the male-sterile line Bcajh97-01A and fertile line Bcajh97-01B of chinese cabbage-pak-choi. (A) Microsporocytes of Bcajh97-01A at anaphase II. (B) Microsporocytes of Bcajh97-01B at anaphase II. (C) Microsporocytes of Bcajh97-01A at telophase II were loose and irregular even though they contained four nuclei. (D) Microsporocytes of Bcajh97-01B at telophase II are enclosed in a thick wall of callose and have concentrated cytoplasm. (E) No tetraspores could be formed in Bcajh97-01A because the intersporal walls were absent in tetrads. (F) The typical cruciate tetraspore of Bcajh97-01B. (G) The grain-like matter in Bcajh97-01A did not enclose granular cytoplasm and a centrally placed nucleus. (H) The smooth round uninucleate microspores of Bcajh97-01B. (I) Pollen sac at late stage in Bcajh97-01A was filled with cytoplasm. (J) Pollen sac of Bcajh97-01B was full of mature pollen grains. (K) Pollen sac of Bcajh97-01A became absolutely empty during the stage before flowering. (L) Pollen sac of Bcajh97-01B at the stage before flowering; $\mathrm{Mp}=$ mature pollen; $\mathrm{Ms}=$ microsporocyte; $\mathrm{N}=$ nuclear; $\mathrm{T}=$ tetraspore; $\mathrm{Tp}=$ tapetum; $\mathrm{Um}=$ uninucleate microspore.
To determine how pollen development was perturbed after telophase II of meiosis in Bcajh97-01 A, we examined the entire pollen development process in both Bcajh97-01A and Bcajh9701B by transmission electron microscopy (TEM). No significant differences were detected at early microspore developmental stages such as the pollen mother cell stage and prophase I between Bcajh97-01A (Fig. 2A-2B) and Bcajh97-01B (Fig. $3 \mathrm{~A}-\mathrm{B})$. The earliest detectable difference was the failure of cytokinesis at the end of male meiosis in Bcajh97-01A. Microsporocytes appeared to proceed normally through karyokinesis of meiosis, except for the sticky chromosome at diakinesis, but intersporal walls were absent in tetrads of Bcajh97-01A (Fig. 2C). Multiple nuclei were apparent within the microsporocyte in most of the TEM pictures.

The most dramatic phenotype showed plasma membranes with varying extents of inward furrowing toward the interior of the syncytium. The invaginations were surrounded with ingrowth callose (Fig. 2D-E). At the late stage, these "microspores" were rife with these near-spherical structures, in which the outer layer was the primexine layer of the pollen wall, developed from the ingrown plasma membrane, and the inside was the expanding callose (Fig. 2F-G). The normal infurrowing of the plasma membrane, which should appear during the meiosis stage, did not occur in the Bcajh97-01A microsporocyte. This defect led to the continuous formation of these round structures. The substances in these abnormal "microspores" subsequently seemed to disperse, and no organelles were observed. Ultimately, the entire "microspore" dissolved into the anther locule (Fig. 2H-I). In the case of the Bcajh9701B microspore, the microsporocyte formed a tetrad after karyokinesis (Fig. 3C) and cytokinesis, and the microspores within a tetrad were individually deposited in the primexine (Fig. 3D). Whereas the Bcajh97-01A "microspore" became severely dispersed, the microspore in Bcajh97-01B developed into uninucleate (Fig. 3E) and then binucleate pollen (Fig. 3F).

IDENTIFICATION OF DIFFERENTIALLY DISPLAYED EXPRESSED SEQUENCE TAGS DURING POLLEN DEVELOPMENT USING CDNAAMPLIFIED FRAGMENT LENGTH POLYMORPHISM. Closed flower buds collected at three different developmental stages (Stage 1, bud diameter less than $1.6 \mathrm{~mm}$; Stage 2, bud diameter about within 1.8 to $2.2 \mathrm{~mm}$; and Stage 3, bud diameter about within 3.2 to $3.6 \mathrm{~mm}$ ) of the two sibling lines (Bcajh97-01A and Bcajh97-01B) were used for cDNA-AFLP analysis. The correlation between bud diameter and pollen developmental stage was estimated based on cytological examination. In the fertile Bcajh97-01B plants, Stage 1 flower buds corresponded to the period preceding meiosis. Stage 2 flower buds represented the tetrad to uninucleate pollen period. Flower buds with the largest diameter (Stage 3 ) corresponded to the period of binucleate to mature pollen formation (L. Huang, unpublished data). The cDNA expression profiles of these buds were determined by PCR selective amplification using 256 independent primer combinations. A section of a typical AFLP gel used in this analysis is shown in Figure 4. Many transcript-derived fragments (TDFs) of genes from the flower buds of sterile Bcajh97-01A and fertile Bcajh97-01B plants displayed different expression patterns that could be divided into nine classes (Table 1). We used " 1 " to denote the presence of a TDF and " 0 " to denote the absence of the same TDF in each type of flower bud. For example, pattern T000001 means that TDFs specifically accumulated in the Bcajh97-01B Stage 3 flower buds, whereas pattern T000101 represents those specifically accumulated in the 


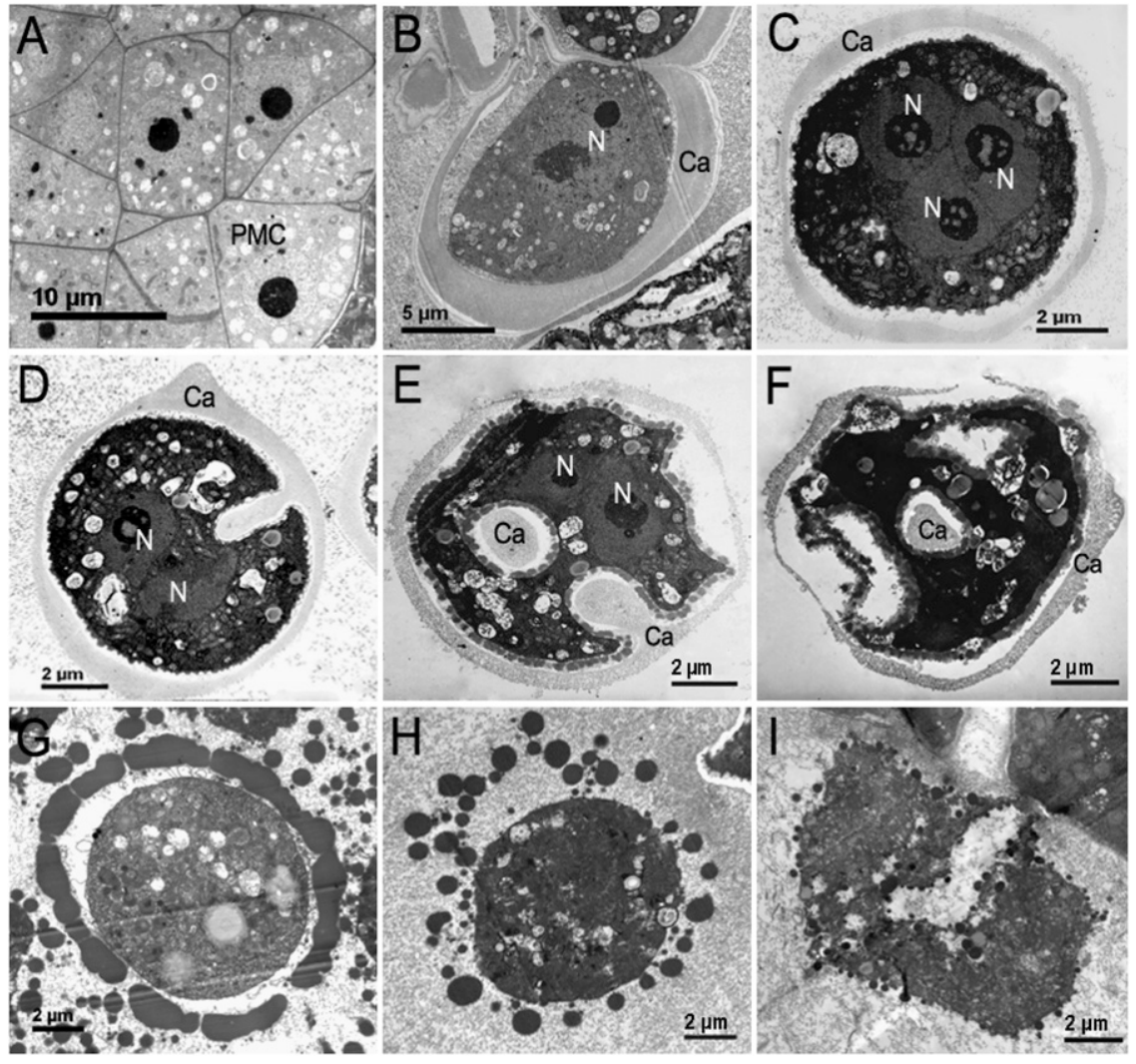

Fig. 2. Transmission electron micrographs of the male-sterile line Bcajh97-01A of chinese cabbage-pak-choi. (AB) The pollen mother cell stage (A) and prophase I (B) seem to be normal. (C) Microsporocyte at telophase II; three microspore nuclei are visible in this section. (D) In-furrowing of the microsporocyte wall and callose. (EF) Formation of a spherical structure, in which the outer layer is the parental plasma membrane and the inside is callose. (G) Several spherical structures in the "microspore" at a late stage. (H-I) The substances in the "microspore" begin to disperse and the "microspore" begins to dissolve; $\mathrm{Ca}=$ callose; $\mathrm{N}=$ nuclear; $\mathrm{PMC}=$ pollen mother cell.

Bcajh97-01B Stage 2 and Stage 3 flower buds. Many TDFs with pattern T010101 or T101010 that specifically accumulated in Bcajh97-01B or Bcajh97-01A flower buds throughout the three stages were identical to the TDFs identified in our previous study of entire inflorescences (Huang et al., 2008c). In addition, six new TDFs were detected that specifically accumulated in the Bcajh97-01B flower buds throughout the three stages. Three others specifically accumulated only in Bcajh97-01A flower buds. Overall, a total of $50 \mathrm{TDFs}$ were differentially displayed, most of which were specifically or predominantly expressed in Bcajh97-01B flower buds, with a smaller number specifically or predominantly expressed in the flower buds of Bcajh97-01A.

In all, 17 TDFs, ranging in length from 100 to $450 \mathrm{bp}$, from six expression patterns, were recovered from gels and reamplified, subcloned, and sequenced for RT-PCR. Of the 17 cDNAs examined, 14 (one specific to Bcajh97-01A flower buds and 13 specific to or predominantly expressed in the flower buds of Bcajh97-01B) had the same expression profiles seen in the original cDNA-AFLP analysis (Table 1; Fig. 5), indicating that the original cDNA-AFLP pattern was validated in $\approx 82 \%$ of cases.

Preliminary identification of the differentially DISPLAYED GENES USING SEQUENCE SIMILARITY SEARCHES. The 14 positive sequences were identified by homology searches using the BLAST program against the GenBank nonredundant public sequence database. Although all of these 14 frag- ments showed close matches (BLASTN and BLASTX expectation values $[E]$ of $<10^{-5}$ ) to database entries (Table 2), most represented genes with unknown or hypothetical functions, similar to those identified in previous genome projects on cruciferous plants (e.g., B. rapa ssp. pekinensis clone $\mathrm{KBrB} 002 \mathrm{G} 19$, Arabidopsis thaliana mRNA sequence, and B. rapa ssp. pekinensis cDNA clone). Only six of the 14 TDFs showed significant homology with known proteins, including the one Bcajh97-01A TDF, which had a T101010 pattern and was designated as BAS3. This TDF shared significant similarities with the $B$. rapa ATP synthase alpha subunit (aptA) gene. Of the five TDFs specifically or predominantly expressed in Bcajh97-01B flower buds, TDF BBS35, with pattern T000101, had a high similarity to A. thaliana polygalacturonase (At3g14060) mRNA. TDF BBS40 and BBS41, with pattern T010101, shared significant homologies with the mRNA of $A$. thaliana lightharvesting chlorophyll a/b-binding protein 2 (Lhcb2) and putative pyrophosphate-dependent phosphofructo-1-kinase, respectively. TDF BBP13, with pattern T110101, was a homolog of the A. thaliana dehydroascorbate reductase (DHAR1) gene. According to known functions, the five genes specifically or predominantly expressed in Bcajh97-01B flower buds can be divided into three classes. One of these genes appears to be associated with pollen wall biosynthesis and regulation, two may play roles in stress responses, and the remaining two are related to metabolism (Table 2).

\section{Discussion}

BCAJH97-01A IS A MUTANT OF MALE MEIOTIC CYTOKINESIS IN CHINESE CABBAGE-PAK-CHOI. In previous morphological and cytological observations, the only difference noted between Bcajh97-01A and Bcajh97-01B was the lack of mature pollen grains in the Bcajh97-01A anther (Huang et al., 2008c). In the present study, the aberrance in pollen development of Bcajh9701A was seen to begin with the failure of intersporal wall formation at the end of meiosis. Cytokinesis of male meiosis was then disturbed after telophase II, whereas other cell cytokinesis appeared to be unaffected. Male sterility in Bcajh97-01A was controlled by a single recessive mutation locus (Huang et al., 2008c), leading us to conclude that Bcajh97-01A is a male meiotic cytokinesis mutant of chinese cabbage-pak-choi controlled by a single locus.

Although a significant number of meiotic mutants have been characterized in various species, especially in maize (Zea mays) (Cande et al., 2009) and the model plant $A$. thaliana (Jiang 

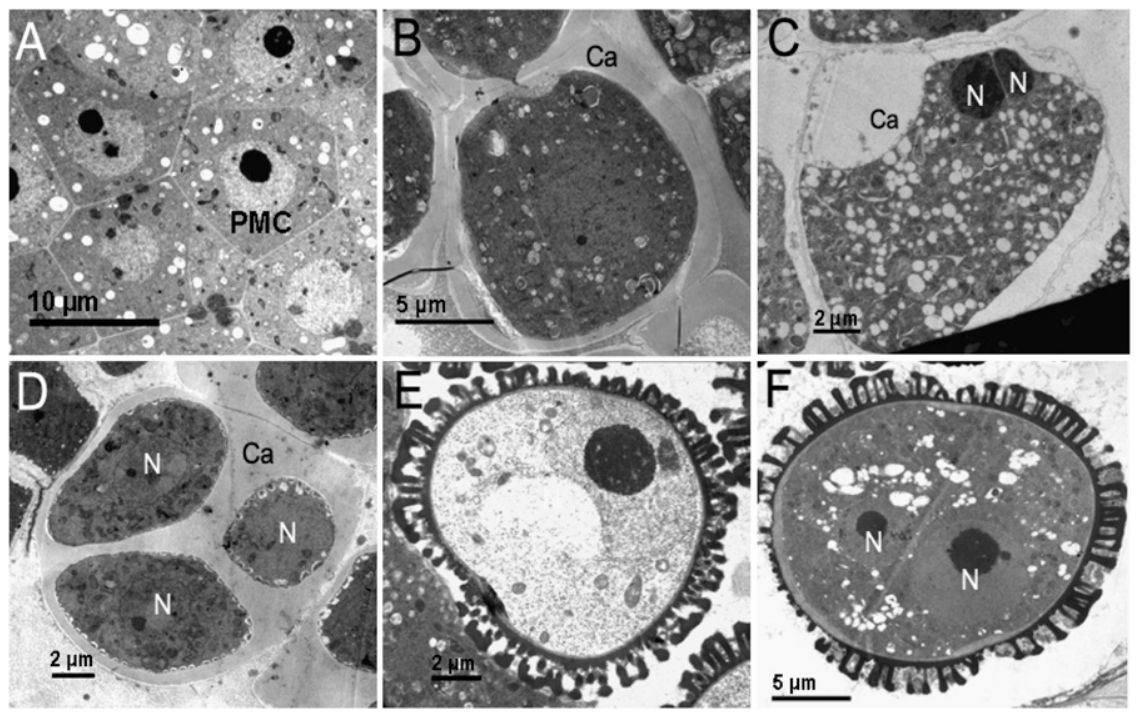

Palmer, 1979; Bione et al., 2002, 2005), and alfalfa (Medicago sativa) (Mariani et al., 2000; McCoy and Smith, 1983). These mutants have effects on other specific cell types (most notably female meiosis and embryo sac development). Another two mutants, tes (Spielman et al., 1997) and std (Hulskamp et al., 1997) from $A$. thaliana, are male meiotic cytokinesis mutants with unaffected female mitosis and meiosis. The particular phenotype of Bcajh97-01A, compared with other male meiotic cytokinesis mutants, is that the plasma membrane stops expanding during its centripetal in-furrowing process and then encircles the callose, giving rise to variously sized spherical structures, which then are immersed in the cytoplasm. At this stage, these "microspores" are irregular and loosened. The whole process is quite The pollen mother cell stage. (B) A microspore at prophase I. (C) A microspore at telophase I. (D) Tetrad stage; three microspores are visible in this section. (E) A microspore at uninucleate stage. (F) A microspore at the binucleate stage; $\mathrm{Ca}=$ callose; $\mathrm{N}=$ nuclear; $\mathrm{PMC}=$ pollen mother cell.

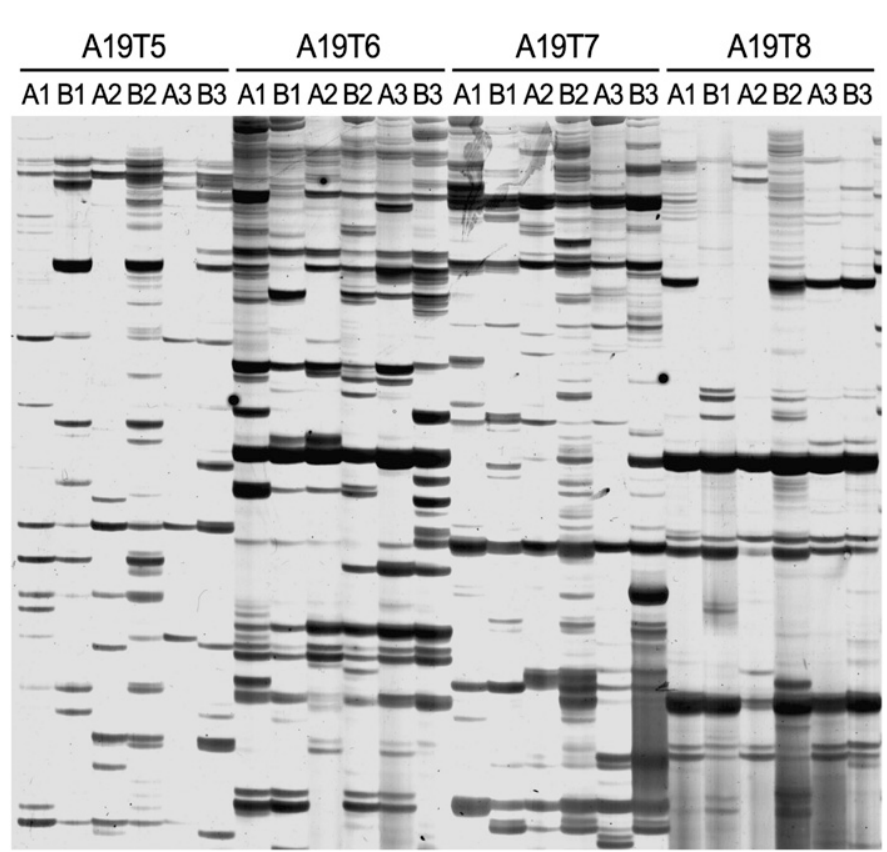

Fig. 4. Accumulation patterns of transcript-derived fragments (TDFs) from the male-sterile line Bcajh97-01A flower buds at three different developmental stages compared with the fertile line Bcajh97-01B of chinese cabbage-pakchoi. Lanes A1, A2, and A3 correspond to the amplified template from the Bcajh97-01A flower buds at Stage 1, Stage 2, and Stage 3, respectively. Lanes B1, B2, and B3 correspond to the amplified template from the Bcajh97-01B flower buds at Stage 1, Stage 2, and Stage 3, respectively. The primer combinations A19T5-8 used are outlined.

et al., 2009; Sanchez-Moran et al., 2005), most are involved in nuclear events with relatively few related to cytokinesis. Failure of male meiotic cytokinesis resulting from a single gene mutation has only been described in a few species, including A. thaliana (Yang et al., 2003b; Zhang et al., 2006), maize (Beadle, 1932), soybean (Glycine max) (Albertsen and similar to that of pinocytosis in plants. Notably, the infurrowing of the plasma membrane does not happen at the same time. The mutant phenotype indicates that the mutated gene encodes for a product necessary for cytokinesis that follows the nuclear divisions of male meiosis but is not required for cytokinesis after either mitosis or female meiosis.

Although mutations affecting mitotic cytokinesis have been previously reported (Assaad et al., 1996; Liu et al., 1997; Lukowitz et al., 1996), Bcajh97-01A is the first mutant reported in chinese cabbage-pak-choi that is directly involved in male meiotic cytokinesis and it provides an excellent source material for study of the molecular mechanism of male sterility.

THE PUTATIVE REASON FOR FAILURE OF CYTOKINESIS IN BCAJH97-01A. The earliest defect detected in the Bcajh97$01 \mathrm{~A}$ pollen is the failure of intersporal wall formation at the end of meiosis, which is similar to the phenotype of tes (Spielman et al., 1997) and std (Hulskamp et al., 1997) mutants in $A$. thaliana. However, the formation of the round callose structures enclosed by plasma membrane is unique to Bcajh97-01 A. In A. thaliana, like in other dicots, microsporocytes divide by simultaneous cytokinesis with intersporal walls forming only after meiosis II (Owen and Makaroff, 1995). Unlike the case in mitotically dividing cells, division sites are not marked by a preprophase band of microtubules. Instead, postmeiotic cleavage planes are most likely determined by "spore domains" defined by arrays of microtubules radiating from each microspore nucleus (Spielman et al., 1997).

Based on the mechanism of cell wall formation after male meiosis suggested by Otegui and Staehelin (2004), we believe that failure of cytokinesis in Bcajh97-01A pollen possibly arises from an incomplete or disorganized array of microtubules in the specialized miniphragmoplasts involved in male meiotic cytokinesis. Incorrect or incomplete microtubule assembly in the central region of the syncytium may block off the in-furrowing of the microsporocyte wall and the fusing of the sheet-like projections in the central region of the syncytium at the conclusion of cytokinesis. Notably, the corresponding gene of the tes mutant, TES, encodes a plant-specific kinesin required 
Table 1. Identification of differentially accumulated transcript-derived fragments (TDFs) in the flower buds of both the male-sterile line Bcajh9701A and fertile line Bcajh97-01B of chinese cabbage-pak-choi at three different developmental stages by cDNA-amplified fragmeng length polymorphism.

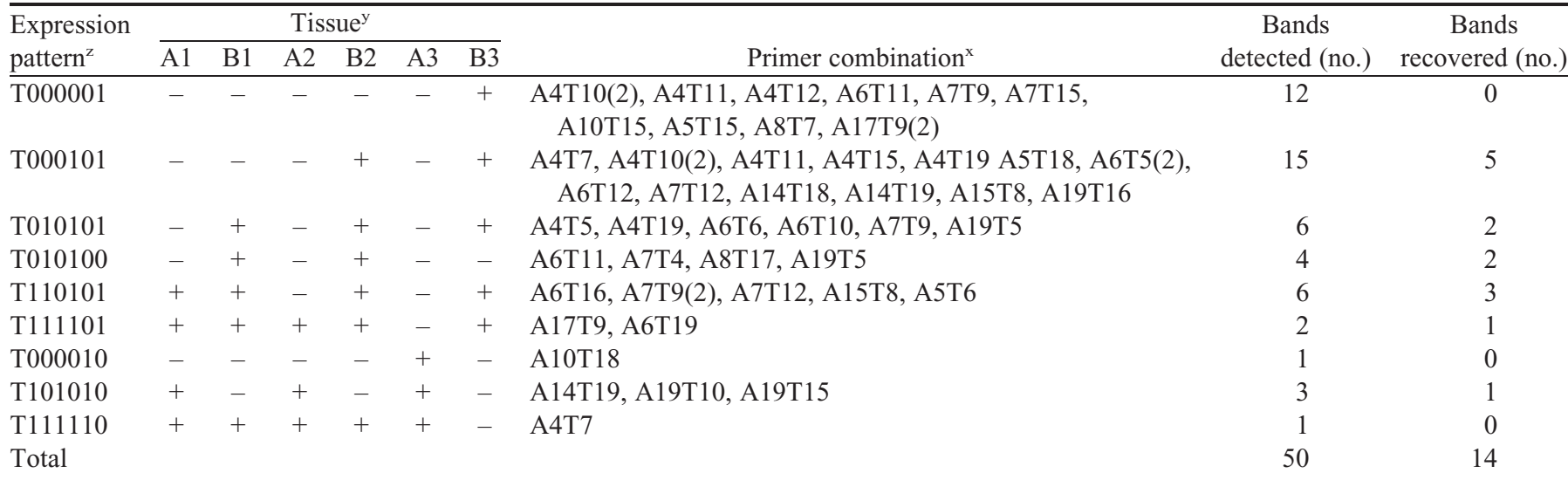

${ }^{\mathrm{z}}$ From left to right, the position of number after the upper case letter T represents each type of flower bud, which are in the following arrangement: Bcajh97-01A Stage 1 flower buds, Bcajh97-01B Stage 1 flower buds, Bcajh97-01A Stage 2 flower buds, Bcajh97-01B Stage 2 flower buds, Bcajh97-01A Stage 3 flower buds, Bcajh97-01B Stage 3 flower buds. Number "1" indicates the presence of a TDF and "0" represent the absence of the same TDF.

${ }^{y}$ A1, A2, and A3 represent Bcajh97-01A flower buds at Stage 1, Stage 2 and Stage 3, respectively. B1, B2, and B3 represent Bcajh97-01B flower buds at Stage 1, Stage 2, and Stage 3, respectively; -/+: band is absent/present.

${ }^{\mathrm{x}}$ The figure in brackets indicates the number of bands derived from this primer combination.

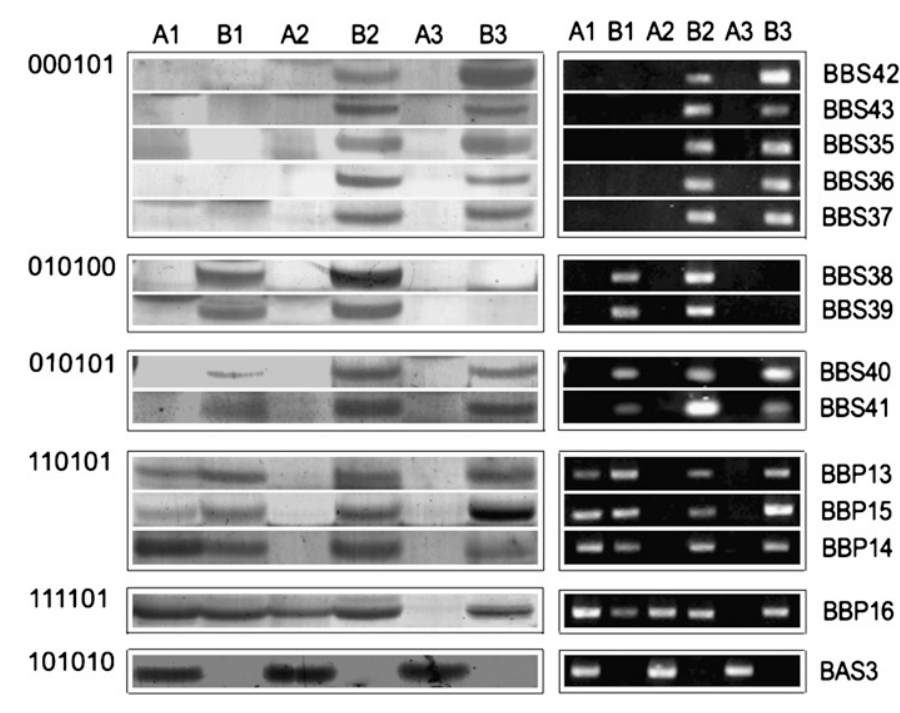

Fig. 5. Reverse transcriptase-polymerase chain reaction (RT-PCR) confirmation of the differential transcript-derived fragments (TDFs) detected by cDNA-amplified fragment length polymorphism (AFLP) between the malesterile line Bcajh97-01A and fertile line Bcajh97-01B of chinese cabbagepak-choi. The left column shows the expression pattern of the TDFs on a cDNA-AFLP gel, and the right column is the corresponding RT-PCR analysis result. Each lane corresponds to the amplified template from the Bcajh97-01A flower buds at three different developmental stages (Lanes A1, A2, and A3) and the Bcajh97-01B flower buds (Lanes B1, B2, and B3). Expression pattern of these TDFs are shown on the left side. From left to right, the position of number after the upper case letter $\mathrm{T}$ represent each type of flower buds, which are in the following arrangement: Bcajh97-01A Stage 1 flower buds, Bcajh97-01B Stage 1 flower buds, Bcajh97-01A Stage 2 flower buds, Bcajh97-01B Stage 2 flower buds, Bcajh97-01A Stage 3 flower buds, Bcajh97-01B Stage 3 flower buds. Number "1" indicates the presence of a TDF and " 0 " indicates the absence of the same TDF. The names of the TDFs are shown on the right side. for male meiotic cytokinesis. The TES protein is likely to function as a microtubule-associated motor, playing a part either in the formation of the radial arrays that establish spore domains after meiosis or in maintaining their stability (Yang et al., 2003a). TEM showed that the defect in cytokinesis in Bcajh97-01A pollen is more severe than that of tes. Positional cloning of the mutant gene in Bcajh97-01A will tell us whether the same gene results in failure of cytokinesis in both the Bcajh97-01A and the tes mutant. Meanwhile, further experiments are needed to address how the plasma membrane is able to enclose the expanding callose.

VARIOUS GENE TYPES MAY BE INVOLVED IN BRASSICA CAMPESTRIS MALE STERILITY. The combination of genetic and transcriptional profiling analysis of mutants has become a powerful tool for dissecting the transcriptional component of the molecular networks underlying cellular functions in eukaryotic organisms (DeRisi et al., 1997). In the basic research field, it has been successfully used to identify genes involved in anther and pollen development. For example, by performing transcript profiling of the $m s 1$ mutant, which codes for a defect in tapetal development and microspore maturation, Yang et al. (2007) identified many genes that are primarily associated with pollen wall and coat formation. However, this tool has rarely been used by agricultural scientists to identify the candidate genes involved in male sterility.

In a previous study, we performed transcriptional profiling of the flower buds from whole inflorescences of both Bcajh9701A and Bcajh97-01B and isolated $51 \mathrm{TDFs}$ that showed some relationship to male sterility (Huang et al., 2008c). Several of these genes have since been characterized and are associated with pollen maturation or pollen wall development (Huang et al., 2008b, 2009a, 2009b; Zhang et al., 2008). However, any particular developmental event in a eukaryote, including pollen development, will be regulated by complex and dynamic changes in gene expression. To construct a framework to 
Table 2. cDNA-amplified fragment length polymorphism differential transcript-derived fragments (TDFs) between the flower buds of both the male-sterile line Bcajh97-01A and fertile line Bcajh97-01B of chinese cabbage-pak-choi at three different developmental stages.

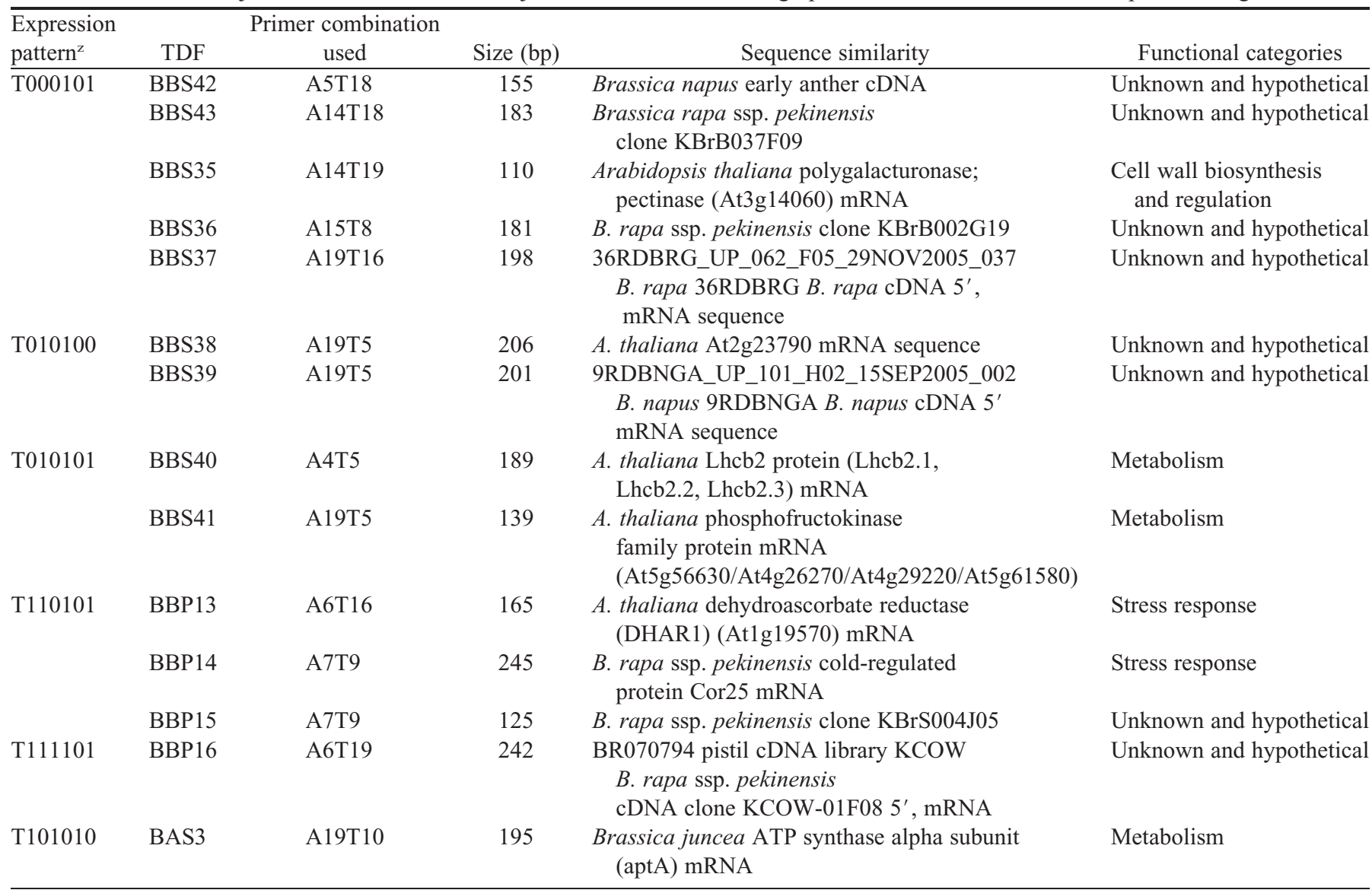

${ }^{\mathrm{z}}$ From left to right, the position of number after the upper case letter T represent each type of flower buds, which are in the following arrangement: Bcajh97-01A Stage 1 flower buds, Bcajh97-01B Stage 1 flower buds, Bcajh97-01A Stage 2 flower buds, Bcajh97-01B Stage 2 flower buds, Bcajh97-01A Stage 3 flower buds, Bcajh97-01B Stage 3 flower buds. Number " 1 " indicates the presence of a TDF and "0" indicates the absence of the same TDF.

describe pollen development at the molecular level, and thereby gain a better understanding of the mechanism of male sterility, it is necessary to investigate gene expression changes during pollen development, like in the present study. As expected, many new TDFs were differentially displayed only at the particular pollen developmental stages that were the focus of this study. In total, 14 new TDFs were found differentially displayed in Bcajh97-01A and Bcajh97-01B flower buds. Eight of these represented genes with unknown or hypothetical functions. Only six of the 14 TDFs showed significant homology with known proteins.

TDF BBS35, which specifically accumulates in the Bcajh97-01B flower buds at Stage 2 and Stage 3, shares a high similarity with the polygalacturonase (PG) At3g14060 mRNA of $A$. thaliana. PG is a cell wall hydrolytic and cell wallloosening enzyme belonging to a large plant gene family and is believed responsible for various cell separation processes. Study of the A. thaliana pollen transcriptome and pollen gene expression in other organisms indicates that there is more than one PG gene expressed in pollen (Allen and Lonsdale, 1993; Hadfield et al., 1998; Honys and Twell, 2003; John and Petersen, 1994; Robert et al., 1993; Tebbutt et al., 1994). During pollen development, many tissue types, including the pollen mother cells, microspores, tapetum, and the septum, undergo cell wall modification (Neelam and Sexton, 1995; Owen and Makaroff, 1995). Thus, PG may play multiple roles in pollen development. In previous studies using whole inflorescences to detect differentially displayed genes in Bcajh97-01A and Bcajh97-01B, we isolated four different PG genes from the flower buds of Bcajh97-01B (Huang et al., 2008c; Wang et al., 2005). When antisense RNA of three of these PG genes was used to transform fertile chinese cabbagepak-choi plants, a decline in male fertility and defects in pollen development and pollen tube growth were observed (Huang et al., 2009a, 2009b; Zhang et al., 2008). The corresponding full-length TDF BBS35 gene has now been cloned (L. Huang, unpublished data) and has been characterized as a new PG gene based on its sequence variations. Consistent with data previously published for $A$. thaliana, maize, and tobacco (Nicotiana tabacum), in which several members of the PG gene family were detected exclusively in pollen (Allen and Lonsdale, 1993; Honys and Twell, 2003), many different PG genes are apparently associated with pollen development. A lack of any of these may be responsible for male sterility.

Another notable newly isolated TDF is BBS40, which is expressed in Bcajh97-01B flower buds throughout the three developmental stages examined in the present study. This TDF shares a high similarity with $A$. thaliana Lhcb2 protein mRNA. 
Lhcb belongs to the light-harvesting chlorophyll $a / b$-binding protein (Lhc) gene family, which shows changes in expression that are required for most of the events choreographed by light. Lhcb2 genes are expressed in A. thaliana mature flowers (Sawchuk et al., 2008), but their expression in pollen has not yet been reported. Because Bcajh97-01A differs from Bcajh9701B only in the failure of pollen formation, we conclude that differences between the Bcajh97-01A and Bcajh97-01B flower bud gene expressions most likely represent differences in pollen gene expression. Thus, the exclusive expression of TDF BBS40 in Bcajh97-01B flower buds and its absence in the flower buds of Bcajh97-01A may indicate a pattern of pollen expression.

We have also identified several other novel gene fragments that are differentially expressed between Bcajh97-01A and Bcajh97-01B flower buds, including TDF BBS41, a candidate homolog of an $A$. thaliana phosphofructokinase family protein and TDF BBP13, which is similar to the A. thaliana dehydroascorbate reductase (DHAR1) gene. The identification of these new fragments supplements currently available information concerning the gene expression profile of pollen as well as suggesting potential candidates underlying the mechanism of plant male sterility.

\section{Literature Cited}

Albertsen, M.C. and R.G. Palmer. 1979. A comparative light-and electron-microscopic study of microsporogenesis in male sterile (MS1) and male fertile soybeans [Glycine $\max (\mathrm{L}$.$) Merr.]. Amer.$ J. Bot. 66:253-265.

Allen, R.L. and D.M. Lonsdale. 1993. Molecular characterisation of one of the maize polygalacturonase gene family members which are expressed during late pollen development. Plant J. 3:261-271.

Araya, A., E. Zabaleta, V. Blanc, D. Brgu, M. Hemould, A. Mouras, and S. Litvak. 1998. RNA editing in plant mitochondria, cytoplasmic male sterility and plant breeding. Electron. J. Biotechnol. 1:31-39.

Assaad, F.F., U. Mayer, G. Wanner, and G. Jurgens. 1996. The KUELE gene is involved in cytokinesis in Arabidopsis thaliana. Mol. Gen. Genet. 253:267-277.

Bachem, C.W.B., R.S. van der Hoeven, S.M. de Bruijn, D. Vreugdenhil, M. Zabeau, and R.G.F. Visser. 1996. Visualization of differential gene expression using a novel method of RNA fingerprinting based on AFLP: Analysis of gene expression during potato tuber development. Plant J. 9:745-753.

Beadle, G.M. 1932. A gene in Zea mays for failure of cytokinesis during meiosis. Cytologia (Tokyo) 3:142-155.

Bione, N.C.P., M.S. Pagliarini, and L.A. De Almeida. 2005. A malesterile mutation in soybean (Glycine max) affecting chromosome arrangement in metaphase plate and cytokinesis. Biocell 29:177-181. Bione, N.C.P., M.S. Pagliarini, L.A. de Almeida, and A.L. Seifert. 2002. An ms 2 male-sterile, female-fertile soybean sharing phenotypic expression with other ms mutants. Plant Breed. 121:307-313.

Cande, W.Z., I. Golubovskaya, C.J.R. Wang, and L. Harper. 2009. Meiotic genes and meiosis in maize, p. 353-375. In: J.L. Bennetzen, and S. Hake (eds.). Handbook of maize: Genetics and genomics. Springer-Verlag, New York, NY.

Cao, J., X. Yu, W. Ye, G. Lu, and X. Xiang. 2006. Functional analysis of a novel male fertility CYP86MF gene in chinese cabbage (Brassica campestris L. ssp. chinensis Makino). Plant Cell Rpt. 24:715-723.

Cao, S. and S. Li. 1980. Breeding and utilization of the chinese cabbage 'Aijiaohuang' genic male-sterile AB line. J. Nanjing Agr. Univ. 1:59-67 [in Chinese].

DeRisi, J.L., V.R. Iyer, and P.O. Brown. 1997. Exploring the metabolic and genetic control of gene expression on a genomic scale. Science 278:680-686
Feng, H., Y. Wei, S. Ji, G. Jin, J. Jin, and W. Dong. 1996. Multiple allele model for genic male sterility in chinese cabbage. Acta Hort. 467:133-142.

Hadfield, K.A., J.K.C. Rose, D.S. Yaver, R.M. Berka, and A.B. Bennett. 1998. Polygalacturonase gene expression in ripe melon fruit supports a role for polygalacturonase in ripening-associated pectin disassembly. Plant Physiol. 117:363-373.

Hanson, M.R. and S. Bentolila. 2004. Interactions of mitocbondria1 and nuclear genes that affect male gametophyte development. Plant Cell 16:154-169.

Honys, D. and D. Twell. 2003. Comparative analysis of the Arabidopsis pollen transcriptome. Plant Physiol. 132:640-652.

Huang, L., J. Cao, A. Zhang, and Y. Ye. 2008a. Characterization of a putative pollen-specific arabinogalactan protein gene, $B c M F 8$, from Brassica campestris ssp. chinensis. Mol. Biol. Rpt. 35:631-639.

Huang, L., J. Cao, A. Zhang, Y. Zhang, and Y. Ye. 2008b. Characterization of $B C M F 10$, a novel gene involved in the pollen wall development of Brassica rapa L. ssp. chinensis Makino. Funct. Plant Biol. 35:1194-1204.

Huang, L., J. Cao, W. Ye, T. Liu, L. Jiang, and Y. Ye. 2008c. Transcriptional differences between the male-sterile mutant bcms and wild-type Brassica campestris ssp.chinensis reveal genes related to pollen development. Plant Biol. 10:342-355.

Huang, L., Y. Ye, Y. Zhang, A. Zhang, T. Liu, and J. Cao. 2009a. $B c M F$ 9, a novel polygalacturonase gene, is required for both Brassica campestris intine and exine formation. Ann. Bot. (Lond.) DOI: $10.1093 / \mathrm{aob} / \mathrm{mcp} 244$.

Huang, L., J. Cao, A. Zhang, Y. Ye, Y. Zhang, and T. Liu. 2009b. The polygalacturonase gene BcMF2 from Brassica campestris is associated with intine development. J. Expt. Bot. 60:301-313.

Huang, L., J. Cao, Y. Zhang, and Y. Ye. 2007. Characterization of a novel gene, BcMF7 that is expressed preferentially in pollen of Brassica campestris L. ssp. chinensis Makino. Sci. China Ser. C: Life Sci. 50:497-504.

Hulskamp, M., N.S. Parekh, P. Grini, K. Schneitz, I. Zimmermann, S.J. Lolle, and R.E. Pruitt. 1997. The STUD gene is required for malespecific cytokinesis after telophase II of meiosis in Arabidopsis thaliana. Dev. Biol. 187:114-124.

Jiang, H., F.F. Wang, Y.T. Wu, X. Zhou, X.Y. Huang, J. Zhu, J.F. Gao, R.B. Dong, K.M. Cao, and Z.N. Yang. 2009. MULTIPOLAR SPINDLE 1 (MPS1), a novel coiled-coil protein of Arabidopsis thatiana, is required for meiotic spindle organization. Plant J. 59:1001-1010.

John, M.E. and M.W. Petersen. 1994. Cotton (Gossypium hirsutum L.) pollen-specific polygalacturonase mRNA: Tissue and temporal specificity of its promoter in transgenic tobacco. Plant Mol. Biol. 26:1989-1993.

Laser, K.D. and N.R. Lersten. 1972. Anatomy and cytology of microsporogenesis in cytoplasmic male sterile angiosperms. Bot. Rev. 38:425-454.

Liu, Z., M.P. Running, and G. Meyerowitz. 1997. TSO1 function in cell division during Arabidopsis flower development. Development 124:665-672.

Lukowitz, W., U. Mayer, and G. Jurgens. 1996. Cytokinesis in Arabidopsis embryo involves the syntaxin-related KNOLLE gene product. Cell 84:61-71.

Mariani, A., P. Campanoni, S. Giani, and D. Breviario. 2000. Meiotic mutants of Medicago sativa show altered levels of alpha- and betatubulin. Genome 43:166-171.

McCoy, T.J. and L.Y. Smith. 1983. Genetics, cytology, and crossing behavior of an alfalfa (Medicago sativa) mutant resulting in failure of the postmeiotic cytokinesis. Can. J. Genet. Cytol. 25:390-397.

Neelam, A. and R. Sexton. 1995. Cellulase (endo-1, 4 glucanase) and cell wall breakdown during anther development in the sweet pea (Lathyrus odoratus L.): Isolation and characterization of partial cDNA clones. J. Plant Physiol. 146:622-628.

Otegui, M.S. and L.A. Staehelin. 2004. 3D Tomographic analysis of post-meiotic cytokinesis during pollen development in Arabidopsis thaliana. Planta 218:501-515. 
Owen, H.A. and C.A. Makaroff. 1995. Ultrastructure of microsporogenesis and microgametogenesis in Arabidopsis thaliana (L.) Heynh. ecotype Wassilewskija (Brassicaceae). Protoplasma 185:7-21.

Robert, L.S., S. Allard, J.L. Gerster, L. Cass, and J. Simmonds. 1993. Isolation and characterization of a polygalacturonase gene highly expressed in Brassica napus pollen. Plant Mol. Biol. 23:1273-1278. Sanchez-Moran, E., R. Mercier, J.D. Higgins, S.J. Armstrong, G.H. Jones, and C.H. Franklin. 2005. A strategy to investigate the plant meiotic proteome. Cytogenet. Genome Res. 109:181-189.

Sawchuk, M.G., T.J. Donner, P. Head, and E. Scarpella. 2008. Unique and overlapping expression patterns among members of photosynthesis-associated nuclear gene families in Arabidopsis. Plant Physiol. 148:1908-1924.

Spielman, M., D. Preuss, F.L. Li, W.E. Browne, R.J. Scott, and H.G. Dickinson. 1997. TETRASPORE is required for male meiotic cytokinesis in Arabidopsis thaliana. Development 124:2645-2657.

Tebbutt, S.J., H.J. Rogers, and D.M. Lonsdale. 1994. Characterization of a tobacco gene encoding a pollen-specific polygalacturonase. Plant Mol. Biol. 25:283-297.

Wang, Y., W. Ye, J. Cao, X. Yu, X. Xiang, and G. Lu. 2005. Cloning and characterization of the microspore development related gene BcMF2 in chinese cabbage pak-choi (Brassica campestris L. ssp. chinensis Makino). J. Integr. Plant Biol. 47:863-872.

Wei, Y., H. Feng, and C. Zhang. 1992. The inheritance of gene male sterility in chinese cabbage (Brassica pekinensis Rupr.). J. Shenyang Agr. Univ. 23:260-266 [in Chinese].
Yang, C., G. Vizcay-Barrena, K. Conner, and Z.A. Wilson. 2007. MALE STERILITY1 is required for tapetal development and pollen wall biosynthesis. Plant Cell 19:3530-3548.

Yang, C.Y., M. Spileman, J.P. Coles, Y. Li, S. Ghelani, V. Bourdon, R.C. Brown, B.E. Lemmon, R.J. Scott, and H.G. Dickinson. 2003a. TETRASPORE encodes a kinesis required for male meiotic cytokinesis in Arabidopsis. Plant J. 34:229-240.

Yang, X., C.A. Makaroff, and H. Ma. 2003b. The Arabidopsis MALE MEIOCYTE DEATH1 gene encodes a PHD-finger protein that is required for male meiosis. Plant Cell 15:1281-1295.

Ye, W., J. Cao, X. Xiang, and G. Zeng. 2003. Molecular cloning and characterization of the genic male sterility related gene CYP86MF in chinese cabbage (Brassica campestris L. ssp. chinensis Makino var. communis Tsen et Lee). J. Hort. Sci. Biotechnol. 78:319-323.

Zhang, Q., L. Huang, T. Liu, X. Yu, and J. Cao. 2008. Functional analysis of a pollen-expressed polygalacturonase gene BcMF6 in chinese cabbage (Brassica campestris L. ssp. chinensis Makino). Plant Cell Rpt. 27:1207-1215.

Zhang, S., Z. Song, and X. Zhao. 1990. Breeding of interactive genic sterile line in chinese cabbage (Brassica pekinensis Rupr) and utilization model. Acta Hort. Sinica. 17:117-125 [in Chinese].

Zhang, W., Y.J. Sun, L. Timofejeva, C.B. Chen, U. Grossniklaus, and H. Ma. 2006. Regulation of Arabidopsis tapetum development and function by dysfunctional tapetum 1 (dyt1) encoding a putative bHLH transcription factor. Development 133:3085-3095. 